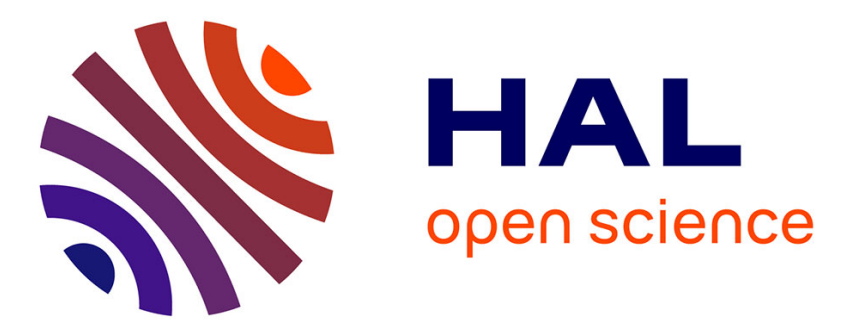

\title{
Propriétés dynamiques d'alliages ferromagnétiques cobalt/métal de transition, faiblement substitués en terres rares magnétiques
}

\author{
J. Russat, G. Suran, H. Ouahmane, M. Rivoire, J. Sztern
}

\section{To cite this version:}

J. Russat, G. Suran, H. Ouahmane, M. Rivoire, J. Sztern. Propriétés dynamiques d'alliages ferromagnétiques cobalt/métal de transition, faiblement substitués en terres rares magnétiques. Journal de Physique IV Proceedings, 1992, 02 (C3), pp.C3-223-C3-227. 10.1051/jp4:1992333 . jpa-00251538

\section{HAL Id: jpa-00251538 https://hal.science/jpa-00251538}

Submitted on 1 Jan 1992

HAL is a multi-disciplinary open access archive for the deposit and dissemination of scientific research documents, whether they are published or not. The documents may come from teaching and research institutions in France or abroad, or from public or private research centers.
L'archive ouverte pluridisciplinaire HAL, est destinée au dépôt et à la diffusion de documents scientifiques de niveau recherche, publiés ou non, émanant des établissements d'enseignement et de recherche français ou étrangers, des laboratoires publics ou privés. 


\title{
Propriétés dynamiques d'alliages ferromagnétiques cobalt/métal de transition, faiblement substitués en terres rares magnétiques
}

\author{
J. RUSSAT, G. SURAN*, H. OUAHMANE*, M. RIVOIRE* et J. SZTERN*
}

Commissariat d l'Energie Atomique, Centre d'Etudes de Bruyères-le-Chatel, BP. 12, 91680 Bruyeres-le-Chatel, France

"Laboratoire de Magnétisme et Matériaux Magnétiques, Centre National de la Recherche Scientifique, 1 place A. Briand, 92195 Meudon cedex, France

\section{ABSTRACT}

Using radio-frequency $(r-f)$ sputtering, we have prepared in presence of a static magnetic field, ferromagnetic thin films of composition $\left(\mathrm{Co}_{93} \mathrm{Zr}_{7}\right)_{100-{ }_{\mathrm{y}}} \mathrm{RE}_{\mathrm{y}}$ ( $\mathrm{RE}=$ $N d, P r, D y, T b . .$. a magnetic rare earth element). The study was performed for small amounts of RE substitution $(0<\mathrm{y}<3)$. These films exhibit attractive soft magnetic properties similar to CoZr alloys: low coercivity $\left(\mathrm{H}_{\mathrm{c}}-1 \mathrm{Oe}\right)$, and a high saturation induction $\left(\mathrm{B}_{\mathrm{s}}>10 \mathrm{kG}\right)$. A low in-plane uniaxial anisotropy $\mathrm{H}_{\mathrm{k}}$ forms, whose magnitude depends upon the composition and the preparation parameters.

Substitution by RE atoms affects essentially the $\mu$ frequency-response. Depending on the nature, the concentration of $\mathrm{RE}$ atoms and on the value of $\mathrm{H}_{\mathrm{k}}$, a high relative rotational permeability $\mu\left(\mu^{\prime} \sim 500-3000\right.$ at frequencies up to $\left.500 \mathrm{MHz}\right)$ was obtained. Also depending on RE and on y, high $\mu^{\prime \prime}\left(\mu^{\prime \prime}-400-3000\right.$ at $v-1$ GHz) distributed over a very broad frequency range $(\Delta \mathrm{v} \sim 500-2500 \mathrm{MHz})$ was measured. Using the Landau-Lifshitz equations for high frequency permeability and the values of the magnetic parameters measured by FMR, experimental spectra could be well approximated.

\section{INTRODUCTION}

Les couches minces amorphes ferromagnétiques préparées à partir de matériaux du type Co$M T$, où $M T$ est un métal de transition non magnétique, présentent des propriétés magnétiques douces - faible champ coercitif $H_{c}$, forte induction à saturation $B_{s}$, faible anisotropie planaire $H_{k}$ et perméabilité élevée jusqu'à quelques centaines de $\mathrm{MHz}$-, attractives pour la fabrication de têtes de enregistrement/lecture intégrées. Par opposition, les couches amorphes du type Co- $T R$, où $T R$ est une terre rare magnétique, présentent des propriétés magnétiques dures, exploitées pour la fabrication de média d'enregistrement magnétique : fort champ coercitif, forte anisotropie perpendiculaire $H_{p}$, faible aimantation perpendiculaire au plan du dépôt.

Dans cette étude, nous avons caractérisé les propriétés magnétiques de matériaux matériaux ferromagnétiques de composition nouvelle $\left(\mathrm{Co}_{100-x-y}-M T_{x}-T R_{y}\right)$ en couches minces. Notre motivation est basée sur la quasi-absence de résultats [1] sur les propriétés magnétiques d'alliages au cobalt, obtenus par ajout de métaux aux influences contradictoires : $M T$ et $T R$. En 
particulier, nous avons cherché à savoir dans quelle mesure les propriétés douces de Co-MT pouvaient être modulées par la présence de $T R$ en faible quantité.

Parmi les métaux de transition utilisables pour obtenir des alliages amorphes Co-MT aux propriétés ferromagnétiques douces, nous avons choisi le zirconium. En effet, la structure amorphe se développe aux faibles taux de substitution $\left(x_{\min } \sim 5 \%\right.$ at.), grâce au fort rayon ionique de $\mathrm{Zr}$, ce qui permet de maximiser l'induction à saturation du matériau.

\section{METHODES EXPERIMENTALES}

Les couches minces amorphes, épaisses d'environ $2000 \AA$, ont été déposées par pulvérisation radio-fréquence sur substrats de verre. Le porte-substrats utilisé comportait une paire d'aimants permanents et un blindage magnétique, afin d'éliminer l'anisotropie uniaxiale perpendiculaire au plan de dépôt et d'induire une anisotropie uniaxiale dans le plan $H_{k}$. Les dépôts réalisés à faible pression d'argon $\left(p_{\mathrm{Ar}}=2 \times 10^{-3}\right.$ Torr) nous ont permis d'obtenir une anisotropie dans le plan pratiquement réversible, dont les valeurs sont minimales après dépôt et peuvent être quasiment annulées par traitement thermomagnétique en champ fixe (appliqué dans le plan de la couche et transversalement à l'aimantation du matériau). La variation de composition des films a été obtenue en disposant des inserts de $4,5 \times 4,5 \mathrm{~mm}^{2}$ de terre rare magnétique sur une cible alliée de $\mathrm{Co}_{93} \mathrm{Zr}_{7}$, chaque insert permettant une variation de $y \sim 0.3$ $\%$ at.

Nous avons mesuré la composition des dépôts à la micro-sonde électronique. Les paramètres de base qui ont été mesurés sont : l'induction à saturation $B_{s}$, le champ coercitif $H_{c}$, le champ d'anisotropie uniaxiale dans le plan de la couche $H_{k}$, le rapport gyromagnétique $\gamma$, la perméabilité relative complexe en fréquence $\mu=\mu^{\prime}-j \mu^{\prime \prime}$, les largeurs de résonance en champ $\Delta H$ ou en fréquence $\Delta v$. Les couches ont été systématiquement caractérisées par des mesures (i) quasi-statiques de $B_{s}, H_{c}$ et $H_{k}$ à l'hystérésigraphe à $50 \mathrm{~Hz}$, (ii) de $B_{s}, H_{k}, \Delta H$ et $\gamma$ au spectromètre de résonance ferromagnétique (FMR) fonctionnant à $v_{\mathrm{FMR}}=9,8 \mathrm{GHz}$ et (iii) de $\mu$ et $\Delta v$ au perméamètre (spectres de perméabilité).

Certains échantillons ont été mesurés au magnétomètre à échantillon vibrant (VSM).

\section{RESULTATS ET DISCUSSION}

Pour les faibles taux de substitution étudiés $(0<y<3 \%$ at.), les propriétés magnétiques statiques ne sont que faiblement perturbées. L'induction à saturation décroit avec $y$ et reste comprise entre : $11<B_{s}<13 \mathrm{kG}$. Dans tous les cas, $H_{c}$ est pratiquement indépendant de $y$ et de la nature de la terre rare. Le champ coercitif demeure faible, avec des valeurs qui restent cantonnées autour de 1 Oe. Par opposition, $H_{k}$ crôt continûment avec le taux de substitution : $H_{k}=30-35$ Oe pour $y=0$ et $80-150$ Oe pour $y=3 \%$ at. Afin de maximiser les valeurs de $\mu^{\prime}$ dans la gamme $0-3 \mathrm{GHz}$, nous avons dû faire subir aux échantillons des traitements thermomagnétiques : traitements thermiques sous vide, avec un champ planaire continu appliqué dans l'axe de difficile aimantation. De cette façon, les échantillons sont positionnés (de manière réversible) dans un état quasi-isotrope ou à faible $H_{k}(5-15 \mathrm{Oe})$.

L'ajout de terre rare induit des pertes magnétiques, caractérisées par la largeur des raies de résonance $\Delta H$ en FMR et $\Delta v$ au perméamètre. Les résultats obtenus montrent que la largeur des raies de résonance dépend de $y$ et la nature de la terre rare employée (figure 1). Ces effets sont dus au caractère fortement relaxant des terres rares magnétiques, même à de très faibles taux d'insertions. 


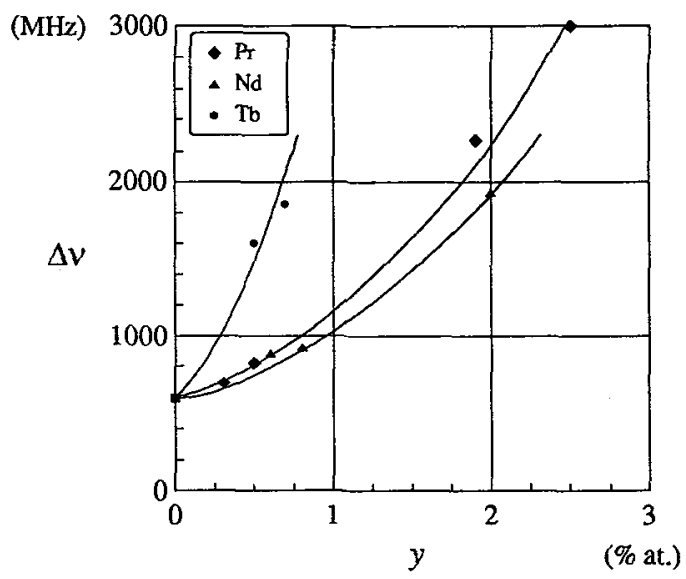

FIG. 1 : Largeur des raies de résonance $\Delta v$ mesurée sur des couches amorphes ferromagnétiques $\left(\mathrm{Co}_{93} \mathrm{Zr}_{7}\right)_{100-}$ ${ }_{y} T R_{y}$, en fonction du taux de substitution en terre rare $y(T R \%$ at.), pour différentes terres rares magnétiques : $y=0$ (matrice $\left.\mathrm{Co}_{93} \mathrm{Zr}_{7}\right), \bullet=\mathrm{Tb}, \boldsymbol{\Delta}=\mathrm{Nd}$ and $\boldsymbol{\nabla}=\mathrm{Pr}$.

La perméabilité, mesurée en statique au VSM (pente des cycles $B-H$ ) et à haute fréquence au perméamètre, dépend de $H_{k}$, TR et $y$. Nous mesurons $500<\mu^{\prime}<2500$ jusqu'à quelques centaines de $\mathrm{MHz}$ et $400<\mu^{\prime \prime}<3000$. La largeur de la raie de résonance est comprise entre : $600<\Delta v<3000 \mathrm{MHz}$ pour une résonance à $1000 \mathrm{MHz}$.

Pour décrire les spectres de perméabilité mesurés, nous avons supposé un mécanisme de rotation cohérente de l'aimantation sous l'action du champ magnétique r-f appliqué dans le plan du film, perpendiculairement à l'axe d'anisotropie. Dans l'équation complète du mouvement de l'aimantation, nous avons utilisé l'expression de l'amortissement de Landau-Lifshitz [2]. L'analyse de l'expression de la perméabilité complexe qui en découle nous permet de relier les largeurs de raies en fréquence $\Delta v$ et en champ $\Delta H(\mathrm{FMR})[3]$ :

$$
\Delta v-\frac{\gamma^{2} \mu_{0} B_{s}}{2 v_{\mathrm{FMR}}} \Delta H
$$

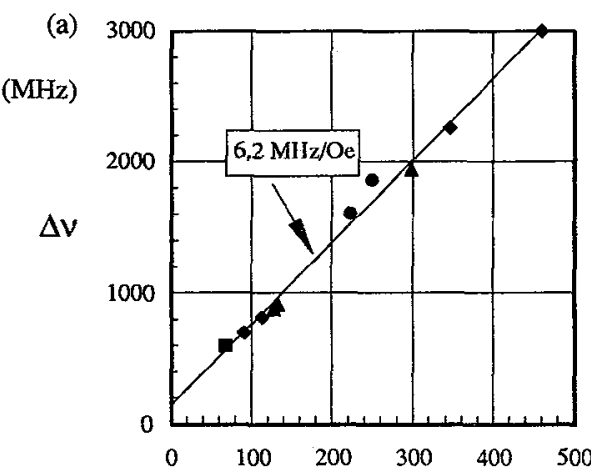

$\Delta H$

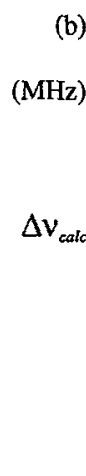

(b)
$\mathrm{MHz})$
$\Delta \mathrm{v}_{\text {calc }}$

(Oe)

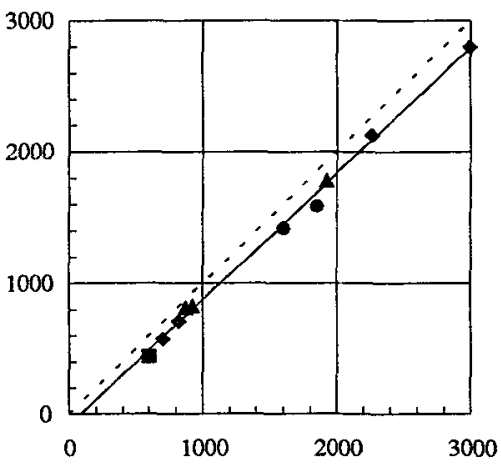

$\Delta \mathrm{v}_{\text {mes }}$

$(\mathrm{MHz})$

FIG. 2 : (a) Relation entre les largeurs de raie expérimentales en fréquence $(\Delta v)$ et en champ $(\Delta H)$; (b) comparaison entre les largeurs de raie théoriques $\left(\Delta v_{c a l c}\right)$ et expêrimentales $\left(\Delta v_{\text {mes }}\right)$. Nous avons utilisé les symboles de la FiG. 1. 
Sur la figure 2 (a), nous observons la dépendance linéaire prévue par l'Eq. (1). La pente de la droite obtenue à partir des données expérimentales est de 6,2 $\pm 0,2 \mathrm{MHz} / \mathrm{Oe}$. La valeur théorique donnée par la figure 2 (b) est de $6,0 \pm 0,1 \mathrm{MHz} / \mathrm{Oe}$.

Les figures 3 (a)-(d) sont des spectres de perméabilité obtenus sur films de différentes compositions. Elles montrent la qualité de l'ajustement du modèle théorique exploitant les paramètres $B_{s}, H_{k}, B_{s}, H_{k}, \Delta H$ et $\gamma$, mesurés en FMR aux données expérimentales. Ces résultats confirment que les mécanismes d'aimantation invoqués plus haut sont bien de nature rotationnelle, avec un amortissement qui dépend de la substitution en $T R$. De plus, la dispersion de l'anisotropie reste négligeable, même pour une aussi forte réduction du champ d'anisotropie par traitement thermomagnétique.
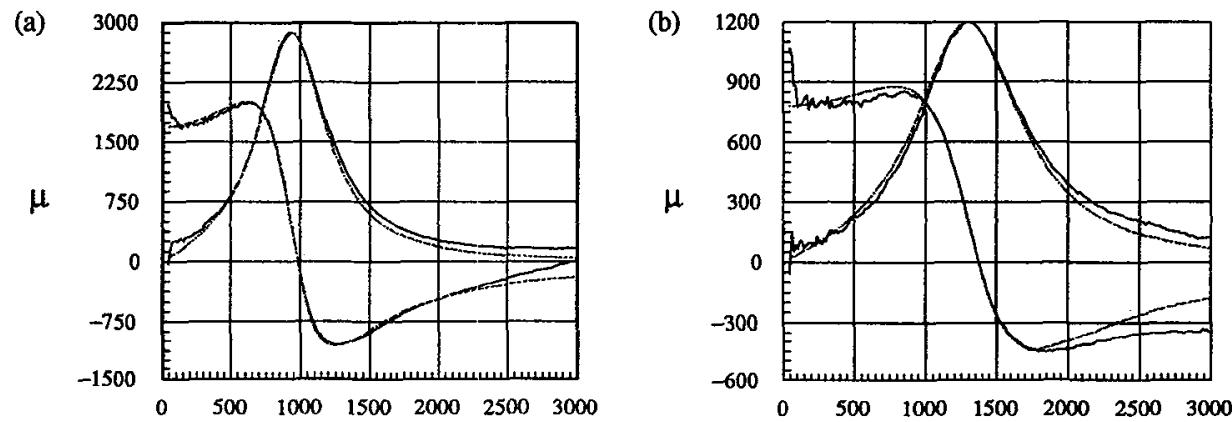

(c)

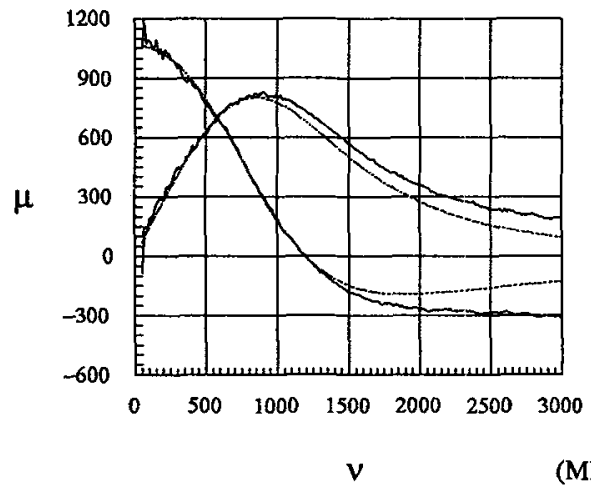

(MHz)

(d)

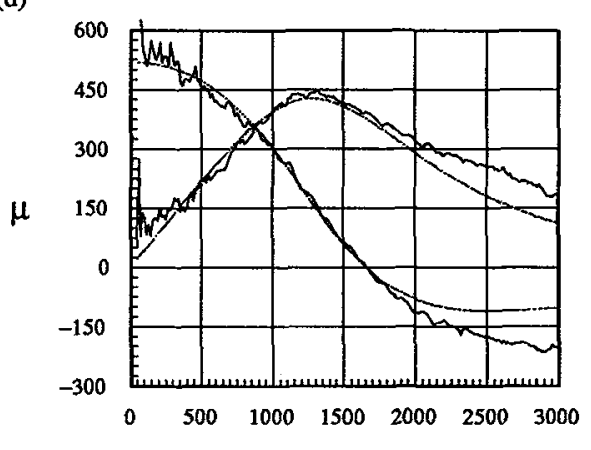

$\checkmark$

(MHz)

FIG. 3 : Spectres de perméabilité complexe expérimentaux (données bruitées) et calculés de couches minces amorphes ferromagnétiques de composition $\left(\mathrm{CO}_{93} \mathrm{Zr}_{7}\right)_{100}-{ }_{y} T R_{y}$. Les différents spectres correspondent aux compositions (a) $y=0$, (b) $y=0,8 \%$ at. Nd, (c) $y=0,7 \%$ at. Tb et (d) $y=1,9 \%$ at. Pr.

\section{CONCLUSION}

Il existe peu de résultats dans la littérature scientifique concernant des matériaux possédant à la fois une forte perméabilité à basse fréquence et des fortes pertes à des fréquences plus élevées. Les résultats les plus convaincants sont ceux rapportés par Walser et al. [4] pour la composition isolée $\mathrm{CO}_{61} \mathrm{~B}_{39}$ après traitement thermomagnétique en champ tournant. A contrario, les caractéristiques magnétiques des matériaux ferromagnétiques réalisés présentent l'inté- 
rêt d'être modulables par le biais de la composition $\left(B_{s}, H_{c}, \mu, \Delta v\right)$ et par les traitements thermomagnétiques postérieurs au dépôt $\left(H_{k}, \mu\right)$.

\section{REFERENCES}

[1] G. Suran, H. Ouahmane et M. Rivoire, J. Magn. Mag. Mat. 125, 104 (1992).

[2] A. H. Morrish, The Physical Principles of Magnetism, (J. Wiley \& sons, NY, 1965).

[3] A. D. Berk, J. Appl. Phys. 28, 190 (1957).

[4] I. S. Jeong et R. M. Walser, IEEE Trans. Magn. MAG 25, 3366 (1989); I. S. Jeong, A. P. Valanju et R. M. Walser, J. Appl. Phys. 64, 5679 (1988). 